\title{
A Model of Heart Rate Kinetics in Response to Exercise
}

\author{
James Robert Stirling ${ }^{a}$, Maria Zakynthinaki ${ }^{b, a}$, Ignacio Refoyo $^{a}$ and Javier Sampedro ${ }^{a}$ \\ ${ }^{a}$ Facultad de Ciencias de la Actividad Física y del Deporte, Universidad Politécnica de Madrid, \\ Avd. Martin Fierro s/n, 28040 Madrid, Spain \\ E-mail: jr.stirling@upm.es \\ ${ }^{b}$ Instituto de Ciencias Matemáticas, CSIC-UAM-UC3M-UCM, c/ Serrano 121, 28006 Madrid, \\ Spain \\ E-mail:mzakyn@mat.csic.es
}

\begin{abstract}
We present a mathematical model, in the form of two coupled ordinary differential equations, for the heart rate kinetics in response to exercise. Our heart rate model is an adaptation of the model of oxygen uptake kinetics of Stirling et al. [21]; a physiological justification for this adaptation, as well as the physiological basis of our heart rate model is provided. We also present the optimal fit of the heart rate model to a set of raw un averaged data for multiple constant intensity exercises for an individual at a particular level of fitness.
\end{abstract}

\section{Introduction (Physiological basis of the model)}

The heart rate, $h r$, is closely related to the rate of change of oxygen uptake, $\dot{V} O_{2}$ : as exercise intensity increases so does the rate of change of oxygen uptake. This rate of increase is determined by the rate at which oxygen is transported to the tissues, the blood's oxygen carrying capacity and the amount of oxygen extracted from the blood. Mathematically the relationship between $\dot{V} O_{2}$ and $h r$ can be expressed [5] in the following way

$$
\dot{V} O_{2}=h r V_{s}(a-v)_{O_{2}}
$$

where

- $V_{s}$ is the amount of blood ejected from the left ventricle per cardiac contraction and

- $(a-v)_{O_{2}}$ is the difference in oxygen content between the coronary arteries and the coronary venous sinus [13].

It should be noted that $h r, V_{s}$ and $(a-v)_{O_{2}}$ are variables which are functions of time and exercise intensity [2,5], hence the relationship between $\dot{V} O_{2}$ and $h r$ is not as simple as it may appear from equation (1.1). The time series of the heart rate can be observed to be geometrically very similar to those of the $\dot{V} O_{2}$ (i.e. approximately exponential), however the kinetics or rates of increase of these two variables for a given exercise intensity are different. 
Due to the similarities in the basic response patterns of the oxygen uptake and heart rate kinetics in response to exercise, we adapt the model of Stirling et al. [21], which was developed to model oxygen uptake kinetics in response to exercise. The tools used in this model are similar to those used by the authors (see $[22,23,24]$ ) to model a variety of other nonlinear phenomena. It should be noted here that the model currently used in the literature to model heart rate kinetics is a curve fitting model, called the 3 (or $n$ ) phase model, after its 3 (or $n$ ) discrete phases (for details regarding the 3 phase model see $[3,6,8,12,14,15,18,21,25,26,27,28])$.

Our model assumes that the heart rate, $h r$, is a function of time $t$ and the intensity $v$ of the particular exercise. The difference between the model we propose here and the one used in [21] is with the function $D(v, t)$. When modelling oxygen uptake kinetics, $D(v, t)$ represents the oxygen demand and, for values of $D(v, t) \geq \dot{V} O_{2_{\max }}$, the excess represents the anaerobic part of the oxygen demand. With heart rate however there is no anaerobic equivalent, hence the function we use does not have the same meaning as in the model presented in [21]. We continue to use a similar mathematical function however as it allows us to model the heart rate kinetics as they approach the maximum heart rate $h r_{\max }$ for intense levels of exercise. In particular it will allow us to model the phenomena whereby the heart rate increases more rapidly to attain the maximum heart rate $h r_{\max }$ the greater the exercise intensity is above the minimum intensity necessary to achieve $h r_{\max }$ [1]. This function $D(v, t)$ will be equivalent to the demand however for values below the maximum heart rate, $h r_{\max }$. In practical terms the function will describe how the exercise intensity effects the heart rate kinetics, and of course it makes sense to think of having exercise intensities greater than the minimum exercise intensity to elicit $h r_{\max }$.

There is always $D(v, t) \geq 0$. We note that there will be $D(v, t) \geq h r_{\max }$ for values of severe or very high intensity exercise and this will result in a peak $h r(v, t)$ equal to $h r_{\max }$, assuming the exercise can be continued for a sufficiently long time period. An exercise at the minimum value of the speed or intensity for which we can achieve $h r_{\max }$ inclusive of the so called slow component $[3,6,8,12,14,15,18,25,26]$ will give $D(v, t)=h r_{\max }$. The value of this speed is found to be slightly (ie. somewhere before the next speed increment) above that of the so called critical speed $[4,10]$. Above the critical speed (i.e. during high intensity exercise) the $h r(v, t)$ is not stabilized and hence the $h r(v, t)$ continues to rise until it reaches the $h r_{\max }$ unless fatigue sets in before $h r_{\max }$ can be achieved.

For exercises where the heart rate demand is such that $D(v, t) \ll h r_{\max }$ it is believed that the value of the demand $D(v, t)$ is, to a good approximation, constant and equal to the end of exercise steady state, $h r_{s s}$, which can be obtained from the time series of the $h r(v, t)$. Note that for higher values of $D(v, t)$ especially for the cases were $D(v, t)>h r_{\max }$ the heart rate demand is probably a function of time. As the intensity of the exercise becomes closer to our absolute speed limit we will become less efficient the longer we exercise at that intensity. This could result in a time dependant behavior in the heart rate demand $D(v, t)$.

\section{Modelling the heart rate kinetics as a dynamical system}

\subsection{The model}

We assume that the rate of change of heart rate $\frac{d}{d t} h r(v, t)$ is a function of the current value of the heart rate, the intensity of the exercise (ie. the speed, $v$ ) and time $t$. This is supported by the observation $[1,14]$ that the amplitude of the rapid part of the heart rate response (the part where there is a steep increase in heart rate before the steady state or slowly increasing part) increases 
more rapidly the heavier the work load imposed. Heart rate is also shown to increase rapidly with virtually no delay at the start of exercise [11, 14]. It is shown, however [7, 14], that for the offtransient response following very intense work loads the heart rate persists at these high values for some seconds before the rapid drop off occurs.

The heart rate as a variable lies always within the physiological limits,

$$
h r_{\min } \leq h r(v, t) \leq h r_{\max } .
$$

We make use of and focus on the physiological fact that, for an exercise demand such that $h r_{\min } \leq D(v, t) \leq h r_{\max }$, the rate of increase of the heart rate kinetics goes to zero on three specific occasions:

- when the heart rate has its minimum value, i.e. when $h r=h r_{\min }$,

- when the heart rate has its maximum value, i.e. when $h r=h r_{\max }$ and

- when the heart rate has reached the heart rate demand of the particular exercise, i.e. when $h r(v, t)=D(v, t)$.

It should be noted here that, when $D(v, t) \geq h r_{\max }$ then $\frac{d}{d t} h r(v, t) \rightarrow 0$ as $h r(v, t) \rightarrow h r_{\text {max }}$.

We denote as $I(t)$ the rate of change of intensity or speed. It is worth noticing that, for linear changes of exercise intensities, $I(t)=c$ where $c$ is a constant, with $c=0$ for the case of constant speed.

Our model for the heart rate kinetics is given by the following system of equations

$$
\begin{aligned}
& \frac{d}{d t} h r(v, t)=A\left[h r(v, t)-h r_{\min }\right]^{B}\left[h r_{\max }-h r(v, t)\right]^{C}[D(v, t)-h r(v, t)]^{E} \\
& \frac{d}{d t} v=I(t)
\end{aligned}
$$

The parameters $A, B, C, E$ in equation (2.1) control the shape of the curve. Parameter $A$ has dimensions of (beats/min $)^{1-B-C-E}$ min $^{-1}$ whilst parameters $B, C$ and $E$ are dimensionless. It is the combined effect of all these parameters which controls the shape of the curve, however the individual parameters mainly effect the following features of the curve:

- Parameter $A$ modifies the magnitude of the rate of change of $h r(v, t)$ and as a result the time taken to reach the steady state value $h r_{s s}$.

- Parameter $B$ controls how quickly we leave or approach the minimum value $h r_{\min }$.

- Parameter $C$ controls how quickly we approach or leave the maximum value $h r_{\max }$.

We note here that $E$ cannot be even or a fraction. If we put $B=C=0$ we get the plateau-type curves that are classically expected below the lactate threshold. $B \neq 0$ and $C \neq 0$ however give a curve which takes much longer to reach a steady state for values of $h r(v, t)$ close to $h r_{\max }$ or $h r_{\min }$. This is very similar to the effect the so called slow component has on the $h r(v, t)$ kinetics.

It is important to remember that all of the parameters $A, B, C$ and $E$ are constants and by definition do not change with exercise intensity hence they give information of fundamental physiological importance for an individual's current level of health and fitness across the continuum of 
exercise intensities and not just for one exercise intensity. When it is understood that health and fitness effect the manner in which we approach the heart rate limits, either during exercise or in recovery, then we can also see the important physiological information that the parameters $B$ and $C$ would give. As one can now easily see there is much physiological information to be found from the parameters of the model presented in equations (2.1) and (2.2).

We note here that in the special case of $v=c$, where $c$ is a constant, then for that particular intensity the heart rate demand is a function of time only, $D(v, t)=D(t)$ and equations (2.1) and (2.2) become uncoupled. This is not the case, however, for time dependent exercise intensities where the coupling of equations (2.1) and (2.2) arises from the fact that the $h r=h r(v, t)$. Furthermore we note that, if we assume that the exercise intensity is constant and sufficiently far from maximal or, for the case of recovery, it does not follow a very heavy exercise period, we can make the usual approximation that $D(v, t)=D$, i.e. the demand $D$ is a constant for a constant velocity, $v=c$.

\subsection{Solution of fixed points}

In a model of coupled ordinary differential equations such as the one given by equations (2.1) and (2.2), fixed points occur when $\frac{d}{d t} h r(v, t)=\frac{d}{d t} v=0$, for $\frac{d}{d t} D(v, t)=0$.

For the conditions we model here there are three solutions:

- $h r(v, t)=h r_{\min }$,

- $h r(v, t)=h r_{\text {max }}$ and

- $h r(v, t)=D(v)$.

We solve for the linear stability of these fixed points to find how it is that orbits nearby behave. This can be used to fix the value of the parameters $A, B, C, E$.

The eigenvalues $\lambda$ of the Jacobian matrix of the system are

$$
\lambda=\frac{\frac{\partial}{\partial h r}\left(\frac{d}{d t} h r\right)+\frac{\partial}{\partial v}\left(\frac{d}{d t} v\right) \pm \sqrt{\left[\frac{\partial}{\partial h r}\left(\frac{d}{d t} h r\right)-\frac{\partial}{\partial v}\left(\frac{d}{d t} v\right)\right]^{2}+4\left[\frac{\partial}{\partial v}\left(\frac{d}{d t} h r\right)\right]\left[\frac{\partial}{\partial h r}\left(\frac{d}{d t} v\right)\right]}}{2}
$$

As however the time derivative of the velocity $v$ is neither a function of $h r$ nor $v$ then there is $\frac{\partial}{\partial h r}\left(\frac{d}{d t} v\right)=0$ and $\frac{\partial}{\partial v}\left(\frac{d}{d t} v\right)=0$ hence

$$
\begin{aligned}
\lambda_{1}= & 0, \\
\lambda_{2}= & A\left[h r(v, t)-h r_{\min }\right]^{B-1}\left[h r_{\max }-h r(v, t)\right]^{C-1} \\
\cdot & {[D(v)-h r(v, t)]^{E-1}[B \alpha(v, t)-C \beta(v, t)-E \gamma(v, t)] }
\end{aligned}
$$

where

$$
\begin{gathered}
\alpha(v, t) \equiv\left[h r_{\max }-h r(v, t)\right][D(v)-h r(v, t)], \\
\beta(v, t) \equiv\left[h r(v, t)-h r_{\min }\right][D(v)-h r(v, t)]
\end{gathered}
$$

and

$$
\gamma(v, t) \equiv\left[h r(v, t)-h r_{\min }\right]\left[h r_{\max }-h r(v, t)\right]
$$


For $B \neq 1, C \neq 1, E \neq 1$ then we have $\lambda_{2}=0$ for our fixed point solutions $h r(v, t)=h r_{\text {min }}$, $h r(v, t)=h r_{\max }$ and $h r(v, t)=D(v)$ respectively. This means that the solutions for the three fixed points in our system would all have $\lambda_{1,2}=0$ and hence they are neutral fixed points. If we decide we need non neutral solutions however we need at least one of $B, C$ and $E$ to be equal to 1 depending on which solutions we need to be attracting. Depending on the data we model however it may be more important to be able to manipulate the vector fields by changing the values of $B, C$ and $E$ than to be concerned with the fact that the three solutions are neutral with $\lambda_{1}=\lambda_{2}=0$. What we choose for the values of the parameters therefore will depend on the data we are modelling.

\subsection{Solutions for $\lambda_{2}$ when $B \neq 1, C \neq 1, E=1$.}

For the data modelled in this paper we fix the parameter $E=1$, as this best fits the data (see also [21]), and hence in this case there is $\lambda_{2}=0$ for $h r(v, t)=h r_{\min }$ and $h r(v, t)=h r_{\max }$, whilst for $h r(v, t)=D(v)$ we have

$$
\lambda_{2}=-A\left[D(v)-h r_{\min }\right]^{B}\left[h r_{\max }-D(v)\right]^{C}
$$

For $A>0$ and exercise intensities where $h r_{\min }<D(v)<h r_{\max }$, equation (2.3) gives us an attracting solution. For exercises of higher intensities, however, where there is $D>h r_{\max }$ the eigenvalue $\lambda_{2}$ becomes a complex number, the real part of which is required to be negative, for the solution to be attracting at $h r(v, t)=D(v)$. Since the real part of $\left[h r_{\max }-D(v)\right]^{C}$ for $D>h r_{\max }$ is

$$
\operatorname{Real}\left\{\left[h r_{\max }-D(v)\right]^{C}\right\}=\left[D(v)-h r_{\max }\right]^{C} \cos (C \pi)
$$

then parameter $C$ has to be within the range

$$
\cos (C \pi)>0 .
$$

\section{Fitting the model to sets of heart rate data}

\subsection{The case where $D(v, t)=D(v)$}

In the work that follows, the heart rate demand is assumed to be a function of velocity only, i.e. $D(v, t)=D(v)$ and for a particular constant velocity $\left(\frac{d}{d t} v=0\right)$ there is $D(v, t)=D=$ constant. We show that this assumption is valid for exercise intensities which are not too close to maximal, and recoveries which do not follow very heavy exercise intensities.

\subsection{Experimental protocol}

The data was collected from a healthy male subject (age 33, height $1.83 \mathrm{~m}$ and weight $82 \mathrm{Kg}$, maximum heart rate $h r_{\max }=185$ beats $/ \mathrm{min}$ and minimum heart rate $h r_{\min }=40$ beats $/ \mathrm{min}$ ). The experiment, which was carried out on a tartan track, followed to as good an approximation as possible, a square wave protocol consisting of five work periods, of four laps each (i.e. 1600 meters), with a speed of $v_{1}^{o n}=13.4 \mathrm{Km} / \mathrm{h}, v_{2}^{o n}=14.4 \mathrm{Km} / \mathrm{h}, v_{3}^{o n}=15.7 \mathrm{Km} / \mathrm{h}, v_{4}^{o n}=17.0 \mathrm{Km} / \mathrm{h}$ and $v_{5}^{o n}=17.9 \mathrm{Km} / h$ respectively. The superscript on refers to the on-transient exercises, the speed of each one of which was assured to be constant, by assuring constant $50 \mathrm{~m}$ split times. 
The numerical values of each exercise speed were measured with the traditional way (recording the time for each $400 \mathrm{~m}$ lap) as well as with the use of a Garmin Forerunner 201 GPS system (Garmin [9]) and a calibrated Polar S625x speed-distance heart rate monitor (Polar [16]). The subsequent static recovery between the work periods lasted 10 minutes, during which the subject lay horizontally and still on the floor $\left(v_{i}^{o f f}=0\right.$, for $i=1, \cdots, 5$ where the subscript off refers to the off-transient recovery period).

The heart rate data were recorded on a beat-to-beat basis using a Polar S810i heart rate monitor (Polar [17]). Care was taken to ensure that the experimental environment was free of any additional electromagnetic signals which can interfere with the signal.

\subsection{Normalized equations}

For simplicity and numerical reasons $h r(v, t)$ was normalized such that $0 \leq h r_{n}(v, t) \leq 1$, ie. $h r_{\text {min }_{n}}=0$ and $h r_{\text {max }_{n}}=1$.

The normalized variable $h r_{n}(v, t)$ is derived from the $h r(v, t)$ as follows

$$
h r_{n}(v, t)=\frac{h r(v, t)-h r_{\min }}{h r_{\max }-h r_{\min }}
$$

Equation (2.1) hence takes the normalized form

$$
\frac{d}{d t} h r_{n}(v, t)=A_{n}\left[h r_{n}(v, t)\right]^{B}\left[1-h r_{n}(v, t)\right]^{C}\left[D_{n}(v, t)-h r_{n}(v, t)\right]^{E}
$$

where $A_{n}$ is the normalized parameter

$$
A_{n} \equiv A\left(h r_{\max }-h r_{\min }\right)^{B+C+E-1},
$$

$D_{n}(v, t)$ is the normalized demand and the other parameters $A, B, C$ and $E$ are as in the nonnormalized case.

\subsection{Fitting the model to the basic response pattern of the data}

If we assume that the values of the parameters $A, B, C$ and $E$ of equation (3.2) are known and so is the value of the heart rate demand $D$ of each data set, then the time series $\left\{h r_{k}^{m}\right\}_{k=0}^{N-1}$ that models the heart rate response are obtained from the integration of equation (3.2) in respect to time $t$. It should be noted here that, in accordance with the results of the analysis of the model presented in $[20,21]$ the value of the parameter $E$ was kept constant and equal to $E=1$ during the modelling process.

In this present study we derived a smooth curve describing the basic response pattern of the data through Fourier low-pass filtering (for a discussion see [21, 27, 28]). The curve described by the points $\left\{h r_{k}^{m}\right\}_{k=0}^{N-1}$ that the model provides is considered to be an optimal fit to the smoothed data curve $\left\{h r_{k}^{f}\right\}_{k=0}^{N-1}$ if the sum of the vertical distances between the two curves (residuals) is minimum. This is an optimization problem where the parameters $A, B$ and $C$ of the model are the variables of the minimization. For the constant exercise intensity case of the present study, where the demand is assumed to be constant, its value $D$ was considered to behave as a parameter and hence also be a variable in the optimization process.

The optimization is described in detail in [28]. The aim was to achieve an optimum fit via appropriately modifying the shape of the curve provided by the model, i.e. via the appropriate 
Table 1 Estimated values of the demand for the data sets.

\begin{tabular}{|c|c|}
\hline Exercise & Demand $D(v)($ beats $/$ min $)$ \\
\hline $1^{\text {on }}$ & 146 \\
\hline $1^{\text {off }}$ & 62 \\
\hline $2^{\text {on }}$ & 156 \\
\hline $2^{\text {off }}$ & 72 \\
\hline $3^{\text {on }}$ & 166 \\
\hline $3^{\text {off }}$ & 72 \\
\hline $4^{\text {on }}$ & 175 \\
\hline $4^{\text {off }}$ & non constant \\
\hline $5^{\text {on }}$ & 177 \\
\hline $5^{\text {off }}$ & non constant \\
\hline
\end{tabular}

changes in the values of the variables $A, B, C$ and the value of constant demand $D$. For the numerical optimization, the stochastic optimization algorithm ALOPEX (see also [29]) was successfully implemented in order to calculate the parameter values of the model that best fit the data sets. The choice of the particular optimization algorithm was based on the algorithm's effectiveness and speed of convergence and, most importantly, on the fact that for the particular optimization process no knowledge is required of the functional dependence of the system on the variables under optimization (i.e. the parameters $A, B$ and $C$ of the model and the heart rate demand $D$ ).

We note here that all numerical computations of the present study were performed by algorithms developed and implemented by the authors, via the use of $\mathrm{C}$ programming language.

\section{Results}

As described in section 3.2, the particular data sets of the present study correspond to exercises of constant velocity, i. e. in our case there is $\frac{d}{d t} v=0$. For the exercise intensities we studied the heart rate demand can be estimated, to a good approximation, to be constant, as the exercise intensity of each data set was constant and not sufficiently high to induce a time dependent demand. Table 1 shows the constant values of the demand $D$ that were obtained using the optimization routine for the on-transient data sets.

It is worth noticing that the values of the demands predicted by the optimization routine were, as expected, identical to the observed asymptotic values of the heart rate time series, for each on-transient data set. Regarding the heart rate demands of the off-transient data sets that follow a sufficiently high on-transient exercise (data sets $4^{\text {off }}$ and $5^{\text {off }}$ ), the analysis was not able to estimate a constant value of demand $D(v)$, especially in the initial stages of the off-transient. The heart rate in these two off-transient data sets is obviously heavily affected by the magnitude of the previous on-transient exercise intensity and therefore the corresponding off-transient heart rate demands can no longer be considered constant (i.e. they are functions of time) for these two data sets.

The optimization routine also revealed the following optimum parameter values:

$$
A_{n}=0.54, B=1.63, C=1.75, E=1.0
$$




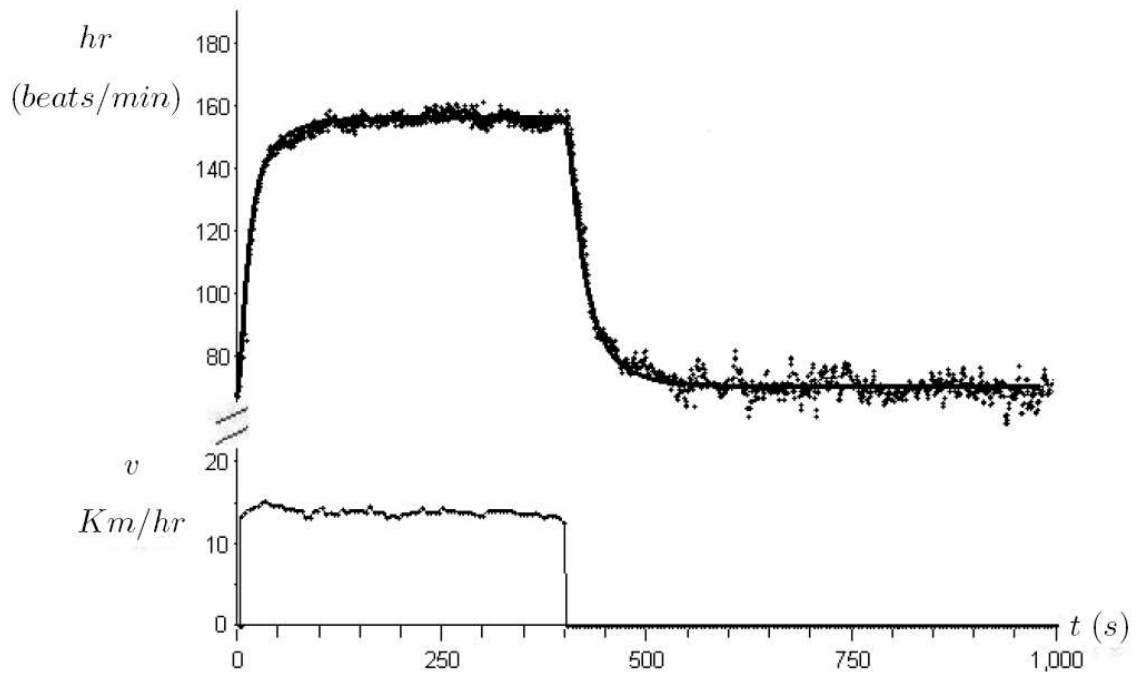

Figure 1. Exercise 2, optimal model fit. 402s on-transient and 600s off-transient.

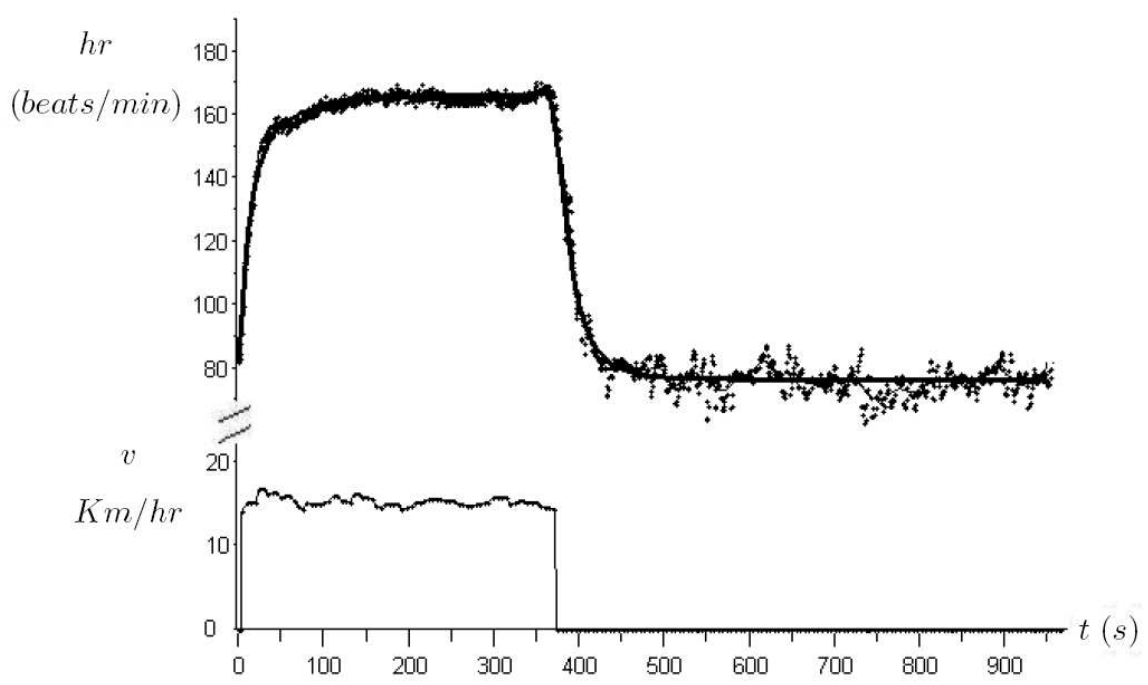

Figure 2. Exercise 3, optimal model fit. 368s on-transient and 600s off-transient.

and for parameter $A$, equation (3.3) gives the value

$$
A=3.217 \cdot 10^{-8}(\text { beats } / \mathrm{min})^{-3.38} \mathrm{~min}^{-1} .
$$

Figures 1,2 and 3 present the model curves (using the optimal parameter values given above) together with the data points. In these figures, the thick curves represent the model and the thin curves are the result of the low-pass filtering of the data. The raw data is plotted as points. In the same graphs the time series of the speed can also be seen.

It can be seen that the parameter values found via the optimization routine provide an excellent fit for the range of demands we have data for and that the model can describe changes in the heart rate kinetics for different exercise intensities by only changing the demand and not the values of 


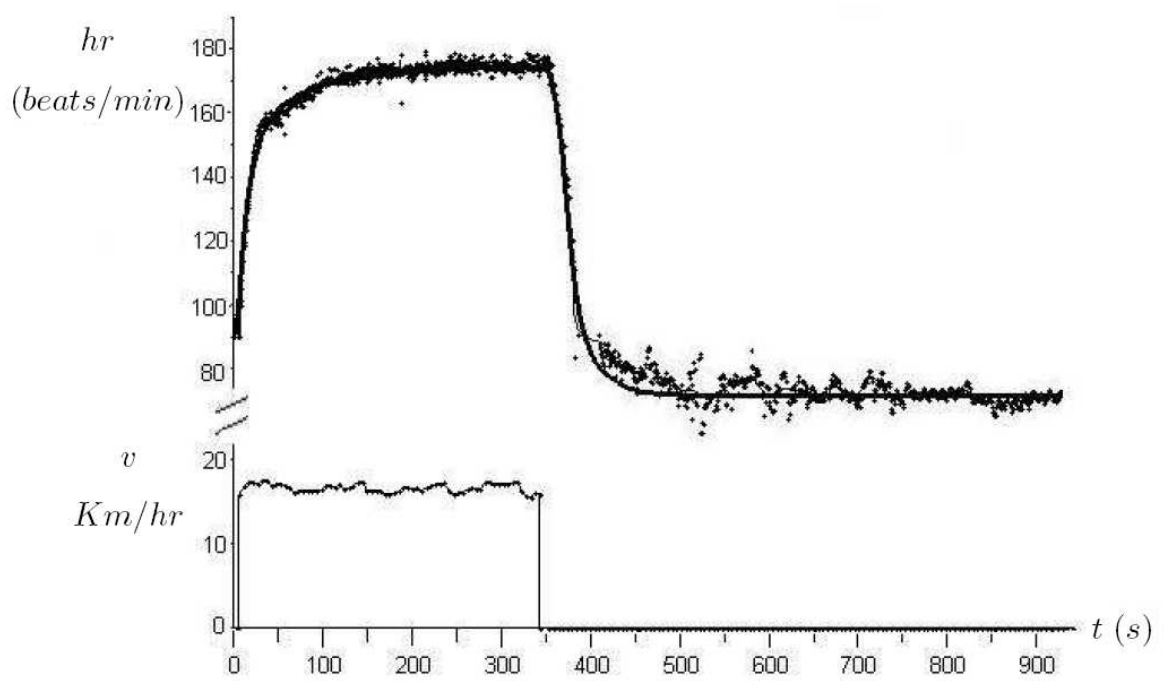

Figure 3. Exercise 4, optimal model fit. 338s on-transient and 600s off-transient.

the parameters. The model's parameters are in fact a characterization of the particular subject and their current fitness level as by definition these parameters will only change with changes in an individuals fitness (see [20] for an example of such a change following a period of training).

In figures 1 and 2 it can be seen that when the on-transient demands are sufficiently low, the standard approximation of a square wave for the demand is reasonable. This assumption breaks down however for higher demands, as can be seen in the off-transient of figure 3. As can clearly be seen in figure 3 , the curve produced by the model for the off-transient does not follow the data in the early stages of the recovery. Initially both the data and the model drop rapidly from the end of exercise heart rate, however the model drops substantially below the data as they approach the new demand. This is because the demand for the model is assumed to be a constant, whilst in reality the demand following the previous heavy bout of exercise has not dropped to the new recovery value sufficiently quick to assume an instantaneous transition between the on-transient demand and the off-transient recovery demand (see also[7, 14]). For an off-transient following a sufficiently high intensity on-transient, the off-transient recovery demand would need to be modelled as a function of time, where the demand changes with time between the final on-transient level of the demand to eventually the off-transient recovery level of the demand. In the case of oxygen uptake it has been shown [19] that following intense or exhaustive exercise the oxygen uptake remains elevated and may not return to resting levels the same day the exercise was carried out, in contrast to the behavior following light exercise.

\section{Conclusions}

By finding the optimal parameter values for an individual and changing only the demand we can model the heart rate kinetics for the different demands we have data for, both on- and off-transient kinetics. This shows that the parameters $A, B, C, E$ as we stated in our model don't change with exercise intensity, see [20] for an example of how the parameters $A, B, C$ and $E$ change following an improvement in fitness levels of the subject after a period of training. This is one of the main 
advantages of our model as the parameters give fundamental information about the condition of the individual subject across the spectrum of exercise intensities, not just a particular exercise intensity.

There exist exercise intensities where the demand $D$ is no longer to a good approximation constant and instead changes with time (i.e. the demand is a function of time, $D(t)$ ). In the data we modelled this was seen to occur for an off-transient following a sufficiency high intensity ontransient, see figure 3. In particular it was seen that in the initial stages the predicted (constant demand) curve drops too early, hence indicating that the demand changes with time. The data shows that, following very high intensity exercise, the demand takes time to reduce from the final on-transient value to the off-transient recovery value, and the assumption that this happens approximately instantaneously is no longer valid for such exercise intensities. Such findings, regarding time-dependent demands, also have important implications in oxygen uptake kinetics, when estimating the oxygen deficit and debt and hence the aerobic and anaerobic energy contributions [21]. The usual approximation that the demand is constant could lead to invalid estimations of the oxygen deficit and debt and hence misleading interpretations regarding the aerobic and anaerobic energy contributions.

Acknowledgments. This work was supported by the programs Ramón y Cajal 2004 and I3 2006, Ministerio de Educación y Ciencia, Spain.

\section{References}

[1] Astrand P O and Saltin B, Oxygen uptake during the first minutes of heavy muscular exercise, J. Appl. Physiol. 16 (6) (1961), 971-976.

[2] Astrand P O, Rodahl K, Dahl H A and Stromme S B, Textbook of Work Physiology: Physiological of Bases of Exercise, Human Kinetics, 2003.

[3] BARstow T J and Mole P A, Linear and nonlinear characteristics of oxygen uptake kinetics during heavy exercise, J. Appl. Physiol. 71 (6) (1991), 2099-2106.

[4] Billat V L, $\dot{V} O_{2}$ slow component and performance in endurance sports, Brit. J. Sports Med. 34 (2) (2000), 83-85.

[5] Brooks G A, FAhey T D, White T P and BALdwin K M, Exercise physiology, human bioenergetics and its applications, 3rd edition, McGraw-Hill, 1999.

[6] Davies C T, Di Prampero P E and Cerretelli P, Kinetics of the cardiac output and respiratory gas exchange during exercise and recovery, J. Appl. Physiol. 32 (1972), 618-625.

[7] Di Prampero P E, Peeters L and Margaria R, Alactic $O_{2}$ debt and lactic acid production after exhausting exercise in man, J. Appl. Physiol. 34 (1973), 628-632.

[8] Engelen M, Porszasz J, Riley M, Wasserman K, Maehar K and Barstow T J, Effects of hypoxic hypoxia on $\mathrm{O}_{2}$ uptake and heart rate kinetics during server exercise, J. Appl. Physiol. 81 (6) (1996), 2500-2508.

[9] GARmin, Garmin Forerunner 201, Personal trainer and owners manual, Garmin Ltd, 2005.

[10] Hill D W and Ferguson C S, A physiological description of critical velocity, Eur. J. Physiol. Occup. Physiol. 79 (3) (1999), 290-293. 
[11] KRogh A and LindhaRd J, The regulation of respiration and circulation during the initial stages of muscular work, J. Physiol. 47 (1913), 112-136.

[12] LAMARRA I, Whipp B J, WARD S A and WASSERman K, Effect of interbreath fluctuations on characterizing exercise gas exchange kinetics, J. Appl. Physiol. 62 (1987), 2003-2012.

[13] Martin D E and Coe P N, Better training for distance runners, 2nd edition, Human Kinetics, 1991.

[14] LinNARSSON D, Dynamics of pulmonary gas exchange and heart rate changes at start and end of exercise, Acta Physiol. Scand. Suppl. 415 (1974), 1-68.

[15] OZYener F, Rossiter H B, WARD S A and Whipp B J, Influence of exercise intensity on the onand off-transient kinetics of pulmonary oxygen uptake in humans, J. Physiol. 53(3) (2001), 891-902.

[16] POLAR, S625x heart rate monitor, user guide, Polar Electro Oy, 2004.

[17] POLAR, S810i heart rate monitor, user guide, Polar Electro Oy, 2005.

[18] Rossiter H B, Howe F A, Ward S A, Kowalchuk J M, Griffiths J R and Whipp B J, Intersample fluctuations in phosphocreatine concentration determined by P-31-magnetic resonance spectroscopy and parameter estimation of metabolic responses to exercise in humans, J. Physiol. 528 (2) (2000), 359-369.

[19] SAltin B, Anaerobic capacity: Past, present and prospective, In: Taylor A, Gollmack P D (Eds.), Biochemistry of Exercise VII, Human Kinetics, Champaign, IL, 1990, p. 387-412.

[20] Stirling J R, Zakynthinaki M S and Billat V L, Modelling and analysis of the effect of training on $\dot{V} O_{2}$ kinetics and anaerobic capacity, Bul. Math. Biol. 70 (5) (2008), 1348-1370.

[21] Stirling J R, Zakynthinaki M S and Saltin B, A model of oxygen uptake kinetics in response to exercise: Including a method for calculating oxygen demand, deficit and debt, Bul. Math. Biol. 67 (5) (2005), 989-1015.

[22] STIRLING J R and ZAKYNTHINAKI M S, Stability and the maintenance of balance following a perturbation from quiet stance, Chaos 14 (1) (2004), 96-105.

[23] STIRLing J R, Nonlinear dynamics, transport and the patchiness of clouds of pollution in an estuarine fluid flow, Discr. Cont. Dyn. Sys. Series B. 3 (2) (2003), 263-284.

[24] StiRling J R, Transport and bifurcation in a non area preserving 2 dimensional map with applications to the discharge of pollution in an estuarine flow, PHYSICA D 144 (2000), 169-193.

[25] Whipp B J and Wasserman K, Oxygen uptake kinetics for various intensities of constant load work. J. Appl. Physiol. 33 (1972), 351-356.

[26] Xing C H, Cochrane J E, Yamamoto Y and Hughson R H, Frequency domain analysis of ventilation and gas exchange kinetics in hypoxic exercise, J. Appl. Physiol. 71 (1991), 2394-2401.

[27] Zakynthinaki M S, Stirling J R, Sillero M, Sampedro J and Refoyo I, Obtaining the basic response pattern of physiological time series data: a comparison of methods, Materials Matematics, $U A B$ (2007).

[28] Zakynthinaki M S and StiRling J R, Stochastic optimization for modelling physiological time series: application to the heart rate response to exercise, Comp. Phys. Commun. 176 (2) (2007), 98108.

[29] ZAKYNTHINAKI M S and SARIDAKIS Y G, Stochastic optimization for a tip-tilt adaptive correcting system, Comp. Phys. Commun. 150 (3) (2003), 274-292. 